\title{
Billion Organisms per Gram
}

National Cancer Institute

\section{Source}

National Cancer Institute. Billion Organisms per Gram. NCI Thesaurus. Code C71192.

A unit of measure of organism content expressed in billions of organisms per unit of mass equal to one gram. 